\title{
On calculation of the neutron charge radius
}

\section{V.A. Karmanov ${ }^{\mathrm{a} *}$}

${ }^{a}$ Lebedev Physical Institute, Leninsky Prospekt 53, 119991 Moscow, Russia

We show that the anomalous quark magnetic moments and relativistic effects in the nucleon wave function result in the correct value of the neutron charge radius.

\section{INTRODUCTION}

The neutron charge radius squared $r_{E n}^{2}$ (average of $r^{2}$ over the neutron charge distribution in coordinate space) is expressed through derivative of the neutron charge form factor $G_{E}^{n}$ :

$$
r_{E n}^{2}=-6\left[\frac{d G_{E}^{n}\left(Q^{2}\right)}{d Q^{2}}\right]_{Q^{2}=0}, \quad G_{E}^{n}=F_{1}^{n}-\frac{Q^{2}}{4 M^{2}} F_{2}^{n},
$$

where $F_{1}^{n}, F_{2}^{n}$ are the form factors determining the neutron electromagnetic vertex:

$$
\bar{u}_{N}^{\prime} \Gamma^{\rho} u_{N}=F_{1}^{n}\left(Q^{2}\right) \bar{u}_{N}^{\prime} \gamma^{\rho} u_{N}+\frac{i F_{2}^{n}\left(Q^{2}\right)}{2 M} \bar{u}_{N}^{\prime} \sigma^{\rho \nu} q_{\nu} u_{N} .
$$

Here $u_{N}, \bar{u}_{N}^{\prime}$ are the nucleon spinors, $M$ is the nucleon mass and $\sigma^{\rho \nu}=\frac{i}{2}\left(\gamma^{\rho} \gamma^{\nu}-\gamma^{\nu} \gamma^{\rho}\right)$. According to (1) , the neutron charge radius is represented as the sum of two contributions:

$$
r_{E n}^{2}=r_{1}^{2}+r_{F o l d y}^{2}, \quad \text { where } \quad r_{1}^{2}=-6\left[\frac{d F_{1}^{n}\left(Q^{2}\right)}{d Q^{2}}\right]_{Q^{2}=0}, \quad r_{\text {Foldy }}^{2}=\frac{3 \mu_{n}}{2 M^{2}},
$$

$\mu_{n}=F_{2}^{n}(0)$ is the neutron magnetic moment. The contribution $r_{\text {Foldy }}^{2}$ is the Foldy term [1], which appears due the generation of the electric field by the (anomalous) neutron magnetic moment because of its zitterbewegung. With the neutron magnetic moment $\mu_{n}=-1.91$ one finds: $r_{\text {Foldy }}^{2}=-0.126 \mathrm{fm}^{2}$. The experimental value [2] $r_{E n}^{2}=-0.113 \pm 0.005 \mathrm{fm}^{2}$ is very close to $r_{F o l d y}^{2}$ and corresponds to $r_{1}^{2}=0.013 \pm 0.005 \mathrm{fm}^{2}$, that is ten times smaller than $r_{\text {Foldy }}^{2}$.

Most of theoretical calculations, on the contrary, give $r_{1}^{2}$ and $r_{\text {Foldy }}^{2}$ to be close to each other in absolute values and of opposite signs, so, because of cancellation in the sum, $r_{E n}^{2}$ is underestimated. In non-relativistic approximation, with the $S U(6)$ symmetric wave function, the neutron charge form factor is identically zero (see, e.g., [3]): $G_{E}^{n}\left(Q^{2}\right) \equiv 0$. To incorporate the relativistic effects, one usually proceeds from this wave function and takes into account the relativistic spins rotations by means of the Melosh matrices. They break the $S U(6)$ symmetry [3] and give a non-zero value of $r_{E n}^{2}$. However, as shown in

\footnotetext{
*e-mails: karmanov@sci.lebedev.ru, karmanov@isn.in2p3.fr
} 
the paper [⿴囗十 , the leading $1 / m^{2}$ correction, calculated in this way ( $m$ is the quark mass), results in $r_{1}^{2}=-r_{F o l d y}^{2}=1 /\left(3 m^{2}\right)$, so, in this approximation the value of $r_{E n}^{2}=r_{1}^{2}+r_{F o l d y}^{2}$ is still zero. Similar result, by a different method, was found in [5]. Beyond the $1 / \mathrm{m}^{2}$ approximation, $r_{1}^{2}$ and $r_{F o l d y}^{2}$ don't cancel each other, that can be seen from their analytical expressions up to the $1 / m^{4}$ :

$$
r_{1}^{2}=\frac{1}{3 m^{2}}-\frac{\epsilon}{18 m^{3}}-\frac{7 \beta^{2}}{54 m^{4}}, r_{\text {Foldy }}^{2}=-\frac{1}{3 m^{2}}+\frac{7 \epsilon}{54 m^{3}}-\frac{\beta^{2}}{27 m^{4}}, r_{E n}^{2}=\frac{2 \epsilon}{27 m^{3}}-\frac{\beta^{2}}{6 m^{4}},(4)
$$

where $\epsilon=M-3 m, \beta$ is the parameter of the oscillator wave function (see eq. (77) below). We found (41) by the methods of explicitly covariant light-front dynamics [6,7]. Since $\epsilon \approx m$ and $\beta / m>1$, one can expect large high order corrections and invalidity of the $1 / m$ decomposition at all. The numerical calculations without any $1 / m^{2}$ approximation [3, 8, 9] don't reveal a large effect and still underestimate $r_{E n}^{2}: r_{E n}^{2}($ theor $) \approx(0.65 \div 0.70) r_{E n}^{2}($ exp $)$. In the paper [10] the value $r_{E n}^{2}=-0.12 \mathrm{fm}^{2}$ close to the experimental one was obtained, in the point form of dynamics, though there is a deviation of the nucleon magnetic moments.

A reliable calculation of the neutron charge radius (as well as of the nucleon e.m. form factors) should incorporate many contributions, such as relativistic effects in the nucleon wave function, its nontrivial spin structure, exchange currents within nucleon, pionic fluctuations of constituent quarks [11], anomalous quark magnetic moments, etc. In this work we will show that the main discrepancy is easily removed, when the anomalous quark magnetic moments together with the relativistic effects are taken into account. The values of the quark magnetic moments are turned out to be close to those found in literature from other fits. Because of approximate and heuristic character of our consideration, this does not exclude other contributions, but shows importance of those which we take into account.

\section{SCALAR COUPLING AND ANOMALOUS QUARK MAGNETIC MOMENTS}

The full basis for the nucleon wave function contains sixteen spin structures [7]. We will take the simplest one: $I(12,3)=\left(\bar{u}_{1} \gamma_{5} U_{c} \bar{u}_{2}\right)\left(\bar{u}_{3} u_{N}\right)=-I(21,3)$ corresponding to scalar coupling'2. Here $\bar{u}_{1,2,3}$ are the (conjugated) quark spinors. The same coupling (but not only it), to construct the nucleon wave function, was used in the paper [9].

We represent the symmetric spin-isospin part of the nucleon wave function in the form:

$$
\psi=I(12,3) \cdot \xi(12,3)+I(23,1) \cdot \xi(23,1)+I(31,2) \cdot \xi(31,2),
$$

where $\xi(12,3)=\left(\xi_{1}^{\dagger} i \tau_{2} \xi_{2}^{\dagger}\right)\left(\xi_{3}^{\dagger} \xi_{N}\right)=-\xi_{21,3}$ is the isospin part with the zero pair isospin, $\xi$ is an isospinor. This wave function has the same non-relativistic limit as the Melosh wave function, but it differs from the Melosh one in the relativistic domain. We assume that in neutron $123=d d u$. Calculating (5) for the particular isospinors $\xi d, d, u$, we get: $\psi_{n}=-I(23,1)+I(31,2)$. To simplify the further calculations, it is convenient to return to the canonical order 123 . Using the Fierz identities, we transform $\psi_{n}$ to the form:

$$
\psi_{n}=-\frac{1}{2}\left(\gamma^{\mu}\right)_{12}\left(\gamma_{\mu} \gamma_{5}\right)_{3 N}+\frac{1}{4}\left(\sigma^{\mu \nu}\right)_{12}\left(\sigma_{\mu \nu} \gamma_{5}\right)_{3 N},
$$

\footnotetext{
${ }^{2}$ Note that the parities of $\bar{u}$ and $U_{c} \bar{u}$ are opposite, hence $\bar{u}_{1} \gamma_{5} U_{c} \bar{u}_{2}$ is a true scalar, just due to $\gamma_{5}$.
} 
where: $\left(\gamma^{\mu}\right)_{12}=\bar{u}_{1} \gamma^{\mu} U_{c} \bar{u}_{2},\left(\gamma_{\mu} \gamma_{5}\right)_{3 N}=\bar{u}_{3} \gamma_{\mu} \gamma_{5} u_{N}$, etc. Note that after Fierz transformation the initial scalar coupling (5) is transformed into the sum of vector and tensor couplings in (6). The total wave function is the product of the spin and the momentum parts: $\Psi_{n}=\psi_{n} \psi_{0}$. For $\psi_{0}$ we chose the oscillator form:

$$
\psi_{0}=N \exp \left(-\mathcal{M}^{2} / 2 \beta^{2}\right),
$$

where $\mathcal{M}^{2}=\left(k_{1}+k_{2}+k_{3}\right)^{2}$ is the invariant mass squared of $3 q$-system, $k_{1,2,3}$ are the on-mass shell quark four-momenta, $N$ is found from the normalization condition [7].

Let us introduce the anomalous quark magnetic moments $\kappa_{q}$, representing the quark e.m. vertex similarly to the nucleon one, eq. (2):

$$
\bar{u}_{q}^{\prime} \Gamma^{\rho} u_{q}=e_{q} \bar{u}_{q}^{\prime} \gamma^{\rho} u_{q}+\frac{i \kappa_{q}}{2 m} \bar{u}_{q}^{\prime} \sigma^{\rho \nu} q_{\nu} u_{q} .
$$

We neglect the dependence of the quark form factors on $Q^{2}$. The nucleon form factors are calculated in the explicitly covariant light-front dynamics [6.7], in the impulse approximation, via plus-component of current, by a program of analytical calculation. The two-loop (five-dimensional) integrals are calculated numerically.

In the $1 / m^{2}$ order the results can be obtained analytically:

$$
r_{1}^{2}=\frac{1}{6 m^{2}}+\frac{5 \kappa_{u}}{3 m^{2}}+\frac{7 \kappa_{d}}{3 m^{2}}, \quad r_{\text {Foldy }}^{2}=-\frac{1}{3 m^{2}}-\frac{\kappa_{u}}{6 m^{2}}+\frac{2 \kappa_{d}}{3 m^{2}} .
$$

For the neutron charge radius we thus find:

$$
r_{E n}^{2}=r_{1}^{2}+r_{\text {Foldy }}^{2}=-\frac{1}{6 m^{2}}+\frac{3 \kappa_{u}}{2 m^{2}}+\frac{3 \kappa_{d}}{m^{2}} .
$$

Note that when $\kappa_{u}=\kappa_{d}=0$, we get $r_{E n}^{2}=-\frac{1}{6 m^{2}}$. So, with the wave function (5) there is no cancellation in the $1 / \mathrm{m}^{2}$ order found in [4] with the Melosh wave function (we remind that both wave functions coincide with each other in non-relativistic limit). This cancellation is a peculiarity of the Melosh wave function.

The fit of the nucleon static properties with $m=340 \mathrm{MeV}, \beta=700 \mathrm{MeV} / \mathrm{c}$ (that corresponds to $\beta^{2} / m^{2} \approx 4,<k>=420 \mathrm{MeV} / \mathrm{c}$ ), by numerical calculation not using the $1 / m$ decomposition, results in following anomalous quark magnetic moments:

$$
\kappa_{u}=0.13, \quad \kappa_{d}=-0.11
$$

With these values we get:

$$
\mu_{n}=-1.92, \mu_{p}=3.09 \text { and } r_{E n}^{2}=-0.125 f^{2}\left(r_{1}^{2}=0.002 f m^{2}, r_{F o l d y}^{2}=-0.127 f^{2}\right) .
$$

The values of $\mu_{n}$ and $r_{E n}^{2}$ are rather close to the experimental ones, $\mu_{p}$ is by $10 \%$ larger. With the wave function (5), incorporating only one spin structure of sixteen, and with a simplest, oscillator parametrization we can hardly pretend to a higher accuracy. Note that the calculation by the approximate formula (10) gives rather close result: $r_{E n}^{2}=$ $-\frac{1}{6 m^{2}}+\left(\frac{3 \kappa_{u}}{2 m^{2}}+\frac{3 \kappa_{d}}{m^{2}}\right)=-0.056+(-0.045) \approx-0.1 \mathrm{fm}^{2}$. From here one can see that half of $r_{E n}^{2}$ results from the relativistic effects in the nucleon wave function (the term $-\frac{1}{6 m^{2}}$ ). Another half results from the quark magnetic moments. 
For comparison, we give $\kappa_{q}$ 's found in other papers: $\kappa_{u}=0.09, \kappa_{d}=-0.15$ [8], $\kappa_{u}=$ $-0.05 \div 0.04, \kappa_{d}=0.01 \div-0.05$ [12], $\kappa_{u}=0.36, \kappa_{d}=-0.18$ [13] Though they differ from each other, these values (except for [12]), as well as the values found in the present work, have a common features: $(i)$ the absolute values of $\kappa_{q}$ 's are of the order of 0.1 ; $(i i)$ $\kappa_{u}$ is positive, $\kappa_{d}$ is negative. These features are enough to reproduce the experimental data, whereas the sensitivity of $r_{E n}^{2}$ to $\kappa_{q}$ is high. Putting $\kappa_{q}=0$ or changing the sign of one or of both $\kappa_{q}$ 's, we obtain the neutron magnetic moment and the charge radius incompatible with experiment (see Table 1).

Table 1

Sensitivity of $\mu_{n}$ and $r_{E n}^{2}$ to $\kappa_{q}$ 's. The best fit: $\kappa_{u}=+0.13, \kappa_{d}=-0.11$

\begin{tabular}{cc|c|cccc}
\hline & & $\kappa_{u}=+0.13$ & $\kappa_{u}=0$ & $\kappa_{u}=-0.13$ & $\kappa_{u}=+0.13$ & $\kappa_{u}=-0.13$ \\
& Exp.: & $\kappa_{d}=-0.11$ & $\kappa_{d}=0$ & $\kappa_{d}=-0.11$ & $\kappa_{d}=+0.11$ & $\kappa_{d}=+0.11$ \\
\hline$\mu_{n}$ & -1.91 & -1.92 & -1.46 & -1.70 & -1.21 & -1.00 \\
$r_{E n}^{2}, \mathrm{fm}^{2}$ & -0.113 & -0.125 & -0.07 & -0.20 & +0.07 & -0.01 \\
\hline
\end{tabular}

We conclude that the anomalous quark magnetic moments (with the values close to already known in literature) and the relativistic effects in the nucleon wave function easily result in the correct neutron charge radius.

I am grateful to L.Ya. Glozman for stimulating discussions attracting my attention to this problem.

\section{REFERENCES}

1. L. Foldy, Rev. Mod. Phys. 30 (1958) 471.

2. S. Kopecky et al., Phys. Rev. Lett. 74 (1995) 2427.

3. F. Cardarelli and S. Simula, Phys. Lett. B 467 (1999) 1; Phys. Rev. C 62 (2000) 065201.

4. N. Isgur, Phys. Rev. Lett. 83 (1999) 272.

5. M. Bawin and S.A. Coon, Phys. Rev. C 60 (1999) 025207.

6. J. Carbonell, B. Desplanques, V.A. Karmanov and J.-F. Mathiot, Phys. Reports, 300 (1998) 215.

7. V.A. Karmanov, Nucl. Phys. A 644 (1998) 165.

8. F. Cardarelli, E. Pace, G. Salmè, S. Simula, Phys. Lett. B 357 (1995) 267.

9. E.F. Suisso, W.R.B. Araújo, T. Frederico, M. Beyer and H.J. Weber, Phys. Lett. B 478 (2000) 86.

10. R.F. Wagenbrunn, S. Boffi, W. Klink, W. Plessas and M. Radici, nucl-th/0010048.

11. L.Ya. Glozman, D.O. Riska, Phys. Lett. B 459 (1999) 49.

12. P.L. Chung, F, Coester, Phys. Rev. D 44 (1991) 229.

13. S. Boffi, P. Demetriou, M. Radici and R.F. Wagenbrunn, Phys. Rev. C 60 (1999) 025206 .

\footnotetext{
${ }^{3}$ In ref. [13], in contrast to eq. (8), the quark charge $e_{q}$ is separated as a factor at the front of both form factors. The original values in [13] are: $\kappa_{u}=\kappa_{d}=0.54$. Multiplying 0.54 by the quark charges $2 / 3$ for $u$ and $-1 / 3$ for $d$, we obtain the above values of $\kappa_{q}$ 's from [13] in the definition (8).
} 